\title{
Oral health promotion programmes during orthodontic treatment beneficial
}

\author{
Abstracted from \\ Gray D, McIntyre G. \\ Does oral health promotion influence the oral hygiene and gingival health of patients undergoing \\ fixed appliance orthodontic treatment? A systematic literature review. J Orthod 2008; 35: 262-269 \\ Address for correspondence: Grant McIntyre, Dundee Dental Hospital and School, Dundee, \\ Tayside, UK. E-mail: grant.mcintyre@nhs.net
}

\section{Question: When individuals undergo orthodontic treatment, are oral health promotion interventions effective in improving their oral hygiene and gingival health?}

Data sources The Cochrane Central Register of Controlled Trials, Medline and Embase databases were searched. A hand search was made of the American Journal of Orthodontics and Dentofacial Orthopaedics, (British) Journal of Orthodontics, European Journal of Orthodontics and Angle Orthodontist, Google Scholar and the reference lists of relevant articles.

Study selection Only randomised controlled trials (RCT) and quasi-randomised controlled clinical trials (CCT), which specifically stated that they assessed reductions in dental plaque levels and/ or gingival bleeding when comparing oral health promotion (OHP) interventions, were included. Trials that involved plaque removal by a professional (except at baseline) or the use of proprietary antiplaque agents were excluded.

Data extraction and synthesis Data extraction was carried out independently by two reviewers. Study quality was assessed for their method of allocation, concealment of allocation, masking of assessment and reporting of withdrawals. Direct comparison between the trials was difficult because of the heterogeneity in the outcome measures between the included studies.

Results Six RCT and quasi-randomised CCT met the inclusion criteria. Positive effects on plaque and/ or gingival health were produced in only four of the included trials. OHP did not result in any detectable difference in two of the included trials. None of the trials that were included produced a negative effect of orthodontic OHP on oral hygiene and gingival health.

Conclusions An OHP programme for people undergoing fixed appliance orthodontic treatment produces a short-term reduction (of up to 5 months) in plaque and improvement in gingival health. No particular OHP method produced a greater short-term benefit to periodontal health during fixed appliance orthodontic treatment. Further studies using appropriate methods and, in particular, r longer followup periods are required.

\section{Commentary}

To state that fixed orthodontic appliances impede optimal oral hygiene in patients is to state the obvious. Yet the obvious often escapes attention in the orthodontic routine and this systematic review of literature on the impact of OHP in fixed orthodontic therapy is therefore very appropriate and thought provoking. The objectives of the review are defined very well, and the stated null hypothesis clearly outlines the parameters that can be quantified. Current research reinforces the association of periodontopathogens with elastomerics, and ATP bioluminescence studies validate the reduced retention of oral bacteria with self-ligated brackets. ${ }^{1}$ The contention that enhanced susceptibility because of individual genetic or environmental variations could allow fixed orthodontic appliances to accelerate the transition of gingivitis to periodontitis is valid for everyone who undergoes this type of treatment. The demographic transition that has resulted in greater numbers of adults receiving orthodontic treatment also brings with it the increased risk of loss of periodontal support, especially in those with predisposing patterns of bone loss. So there is absolutely no debate on the issue of the need for OHP in parallel with fixed orthodontic therapy.

General OHP focuses on reducing periodontal disease and, as correctly observed by the authors, OHP efforts in orthodontic patients up to this point have focussed on potential demineralisation. The effects of OHP on the gingival health of orthodontic patients are neither well-documented nor understood.

The search strategy of the review is comprehensive and efforts to remove bias in the literature search, study selection, data abstraction and synthesis are robust. The inclusion criteria yielded a possible 218 studies, and a stringent evidence-based process reduced the studies for inclusion to only six. Herein lies the challenge for the authors, to apply established evidence-based methods to extract data: there is great variability in the study design. None of the included studies have described allocation concealment. Three studies describe blind outcome assessment, and two include withdrawal rates. The authors have done well to state the inter-rater reliability of the methodological quality of trials in relation to randomisation, concealment, blinding and withdrawals. Direct comparison of the trials is difficult because of the heterogeneity of the outcomes measured, which are diverse. The unweighted kappa statistics seem to be the only method to derive a correlation between these disparate measures. An interesting observation that there was no negative effect of OHP on gingival health may seem to be only a passing remark, but serves to reinforce the outcome that such promotion might lead to 
ORTHODONTICS

significant reductions in plaque levels in the short-term for orthodontic patients for whom OHP measures have been taken.

The value of this literature review, which is exhaustive, lies in its very clear clinical bottom-line, although its message is based only on data from six studies. OHP should be integral to each and every orthodontic fixed appliance case, and the patient should receive this material before the commencement of treatment. The evidence here shows a reduction in plaque levels that would not only have a positive effect on the periodontal support but also reduce the acid challenge to enamel, which causes demineralisation and white spots. Clinicians need to adopt strategies to minimise plaque buildup when using orthodontic appliances, but also need to review newer appliances, bracket profile and the benefits derived from self-ligation from the perspective of improved oral hygiene and gingival health. It may seem utopian, but would the product developers factor this concept into the design and promotion of orthodontic appliances?

The obvious question that follows is over which method of OHP is optimal for orthodontics. That is a question for which the authors here were unable to provide answers, based on their systematic review. They have pointed out how videodata might be more effective than written material, and how harnessing newer technology such as podcasts and OHP videos played on a loop in waiting areas could have a very strong positive influence on oral health. Yet the need for each orthodontic practice to develop its own OHP material is paramount. The reviewers were not able to identify a single reliable method, but the services of a professional educator with a plaque disclosing programme would clearly have benefits.

There is also a very important diagnostic issue that emerges from this review. Clinicians need to identify individuals who have existing periodontal bone loss and those with a predisposition to periodontal bone loss, such as the immunosuppressed, diabetics, menopausal and pregnant women, and people with special needs. The effects of dietary modification and smoking need to be amplified in such or most cases and therefore the development of OHP would become a developmental activity for the clinician or the practice, based on the patient profile.

This review is limited by the paucity of valid studies for inclusion, the heterogeneity of available data, and the inability of the reviewers to synthesise the available data. The review still manages to produce the strong message that, although the results of these studies do not show long-term benefits conferred by OHP on the periodontal health of orthodontic patients, the short-term derived benefits are clear: all people who undergo fixed orthodontic therapy should receive OHP from either a hygienist or therapist before and during orthodontic treatment, irrespective of the effectiveness of plaque control at the start of treatment.

There will also be a need to modify the OHP programme/message to suit the individual and practice profiles. The ability to focus on the long-term value of improved oral hygiene and periodontal health could well be the basis for a clinician to view newer brackets or treatment adjuncts from the point of plaque retention. Clinicians would also have to schedule regular oral hygiene sessions for susceptible patients periodically during the entire duration of treatment.

The systematic review brings alive an important issue and lends tremendous credence to evidence-based methods: the latter create a new focus on patient welfare and, after all, the entire purpose of our work is to do good and no harm. The reviewers should be complemented for their work and for defining this real-life clinical bottom line.

Anmol S Kalha

Institute of Dental Studies and Technologies, Modinagar, India

1. Pellegrini P, Sauerwein R, Finlayson $T$, et al. Plaque retention by self-ligating vs elastomeric orthodontic brackets: quantitative comparison of oral bacteria and detection with adenosine triphosphate-driven bioluminescence. Am J Orthod Dentofac Orthopedics 2009; 135: 426.e1-426.e9.

Evidence-Based Dentistry (2009) 10, 111-112. doi:10.1038/sj.ebd.6400686 